Dieses Dokument ist eine Zweitveröffentlichung (Postprint) /

This is a self-archiving document (accepted version):

Stefan Horlacher

'Mothers Reign Supreme'? Weiblichkeit, Mutterschaft und die Wiederkehr des Abjekts in der elisabethanisch-jakobäischen Tragödie: John Webster

Erstveröffentlichung in / First published in:

GeschlechterSpielRäume. Leiden/Boston: Brill | Rodopi 2015, S. 21-42. ISBN 978-90-420-3276-7. DOI: https://doi.org/10.1163/9789042032767 003

Diese Version ist verfügbar / This version is available on: 


\section{'Mothers Reign Supreme'? Weiblichkeit, Mutterschaft und die Wiederkehr des Abjekts in der elisabethanisch-jakobäischen Tragödie: John Webster}

Drawing on the work of Julia Kristeva, Luce Irigaray, Hélène Cixous and media philosopher Jean Baudrillard, this article concentrates on John Webster's tragedies and - after a short discussion of Shakespearean tragedies as well as of The White Devil - argues that The Duchess of Malfi is a unique English Renaissance tragedy insofar as it presents an unprecedented conception of feminine identity, which is linked to an affirmative understanding of female sexuality, to a non-traditional understanding of motherhood and to the notion of the abject. As a consequence of this, the supposed irregularities or 'flaws' of the play can be explained as the semiotic pulsation of drives interfering with the symbolic order, while the play in its totality demonstrates that it is the negated "peculiar organisation of abjection which actually founds the signifying economy of our culture" (A. Smith).

"Die Tatsache, dass aller Sinn aus einer körperlichen und damit sterblichen Basis hervorgeht und in dieser verankert bleibt, dass der mütterliche Körper 'eine seltsame Falte ist, die die Kultur in Natur abändert, das Sprechende in Biologie', eine 'Falte-und-Katastrophe des 'Seins' [. . .], kann in das Symbolische nicht integriert werden"1 - und vielleicht ist genau dies der uneingestandene Grund dafür, dass Mütter (nicht nur) in der englischen Renaissancetragödie selten anders als in zugerichtet-stereotypisierter Form ins Zentrum der Stücke rücken, existiert die Mutter doch in der Regel als ihre Kinder liebende und sich opfernde, als für ihren Nachwuchs planende, vielleicht auch intrigierende, als ihn potentiell verschlingende, als um ihn trauernde oder als ihn rächende Frau. Dass das Motiv der Mutterschaft vom Text nicht stereotyp und analog zu Männerphantasien und -ängsten einschränkendlimitierend funktionalisiert, sondern als bejahender Ausdruck und Symbol einer aktiv und bewusst gelebten weiblichen Sexualität, vielleicht auch spezifisch weiblichen Libido und Identitätskonstruktion propagiert und als eine eigene, dem Patriarchat und der symbolischen Ordnung entgegengesetzte semiotische Kraft aufgefasst, vielleicht sogar gefeiert wird, ist sicherlich die Ausnahme. Wobei es bei den folgenden Überlegungen nicht primär um biologische Mutterschaft geht, sondern vielmehr um ihren Symbolwert für eine im Sinne Julia Kristevas nicht vollständig in das Symbolische integrierbare

\footnotetext{
${ }^{1}$ Inge Suchsland: Julia Kristeva zur Einführung. Hamburg: Junius 1992. S. 147.
} 
Weiblichkeit ${ }^{2}$ sowie die aus dieser Differenzqualität resultierenden Möglichkeiten für alternative Identitäts-, Lebens- und Gesellschaftsentwürfe.

Wie ein Blick auf Shakespeares Tragödien verrät, zeichnen sich viele Heldinnen der englischen Renaissancetragödie gerade nicht primär durch die soeben beschriebene affirmativ-semiotische Form von Weiblichkeit, geschweige denn Mütterlichkeit aus: Lady Macbeth, Goneril und Reagan oder Tamora, Queen of the Goths, um nur einige zu nennen, vermögen kaum Empathie zu wecken oder etwa Gegenentwürfe zum Patriarchat aufzuzeigen. Vielmehr werden sie als machtbewusste und kühl-kalkulierende phallische Frauen porträtiert, die häufig nicht das Ende der Theaterstücke erleben. So muss Tamora, die rächende Mutter, vor ihrem Tod eine Pastete, hergestellt aus dem Fleisch ihrer Söhne Chiron und Demetrius, verspeisen - in der Verfilmung von Julie Taymor anspielungsreich zubereitet und serviert von dem ohnehin mit der Aura des kannibalischen Verbrechers aus The Silence of the Lambs (1991) umgebenen Anthony Hopkins -, und Lady Macbeth - vom Ausmaß ihrer Gräueltaten, aber auch von ihrer Inversion der Geschlechterrollen heimgesucht ${ }^{3}$ - erlebt eine Wandlung von:

Come, you spirits

That tend on mortal thoughts, unsex me here,

And fill me from the crown to the toe, top-full

Of direst cruelty. ${ }^{4}$

$\mathrm{zu}:$

[...] unnatural deeds

Do breed unnatural troubles; infected minds

To their deaf pillows will discharge their secrets:

More needs she the divine than the physician -5

In der Lage, eine spezifisch weibliche oder zumindest von der herrschenden patriarchalen Ordnung differierende Sphäre zu etablieren oder über einen längeren Zeitraum aufrechtzuerhalten, sind diese Frauen in der Regel nicht. Eher im Gegenteil: In Othello wird die anfangs gerade in Bezug auf ihre Eltern, aber auch auf Othello so durchsetzungsfähige Desdemona in ihrem eigenen Schlafzimmer - einem zumindest traditionell feminin codierten

\footnotetext{
${ }^{2}$ Auch die Begriffe "Männlichkeit", "Weiblichkeit" bzw. "männlich" und "weiblich" werden im Folgenden nicht biologisch, sondern im Sinne Kristevas benutzt.

${ }^{3}$ William Shakespeare: Macbeth. I.5,37-53, 70ff.; ebd. I.7,47-58.

${ }^{4}$ Ebd. I.5,39-42.

${ }^{5}$ Ebd. V.1,69-72.
} 
Raum - erdrosselt, ${ }^{6}$ in Hamlet bleibt Gertrude in einer Männerwelt zwischen dem toten König, ihrem neuen Ehemann Claudius und Hamlet gefangen, und auch der ohnehin schwächelnden Vatertochter Ophelia - "No, my good lord, but as you did command"7 - bleibt, nachdem ihr bereits Hamlet mehrfach den Gang ins Kloster nahegelegt hat - "Get thee to a nunnery. [. . .] To a nunnery, go"8 -, als eigener femininer Raum im Schloss von Elsinore nur der Wahnsinn, der Gesang und das Wasser. ${ }^{9}$

Deutlich andere, für ihre Zeit bemerkenswerte, wenn nicht sogar einzigartige Konzeptionen starker Weiblichkeit wie auch der Rolle der Mutterschaft weisen die im Vergleich zum Euvre Shakespeares von der Kritik noch immer vernachlässigten Tragödien John Websters auf, ${ }^{10}$ wobei auch hier differenziert werden muss: So bietet Websters 1612 veröffentlichte und auf der Geschichte der am 22. Dezember 1585 ermordeten Vittoria Accoramboni basierende Rachetragödie The White Devil ${ }^{11}$ zwar eine der bedeutendsten Frauenrollen des englischen Renaissancetheaters, doch bleibt die Protagonistin Vittoria aufgrund ihrer moralisch prekären Situation zumindest ambivalent: Aufgrund ihres Traumes lässt ihr Geliebter, der Herzog von Brachiano, sowohl Vittorias Ehemann Camillo als auch seine eigene Ehefrau, Isabella, ermorden. Als Kardinal Monticelso Vittoria der Mittäterschaft an der Ermordung Camillos sowie des Ehebruchs anklagt, kommt es in der berühmten, mit "The Arraignment of Vittoria" überschriebenen Gerichtsszene zu einem bemerkenswerten showdown, in dem Vittoria eloquent und furchtlos die Machenschaften des Patriarchats entlarvt. Da sie jedoch als Anstifterin des Doppelmordes angesehen werden kann, bleibt ihre Position moralisch unklar, was nicht zuletzt durch die dem Kardinal (und späteren Papst) wie auch Vittoria abwechselnd zugeschriebene Farbsymbolik von Weiß und Scharlachrot ebenso verdeutlicht wird wie durch die Tatsache, dass die Protagonistin gefangengehalten, exkommuniziert und später erstochen wird. Ungeachtet dieser Tatsachen ragt

${ }^{6}$ Zwar teilt Desdemona die Todesart mit der Herzogin von Malfi, doch hat ihre Hochzeit mit Othello vielleicht gesellschaftspolitische, zu keiner Zeit aber die gleiche staatspolitische Bedeutung wie die Hochzeit der Herzogin.

${ }^{7}$ Shakespeare: Hamlet. II.1,109.

${ }^{8}$ Ebd. III.1,122, 150.

${ }^{9}$ Ebd. IV.7,150-155.

${ }^{10}$ Auf Websters city comedies, aber auch auf Theaterstücke wie The Devil's Law Case oder Appius and Virginia kann schon aus gattungstechnischen Gründen nicht eingegangen werden.

${ }^{11}$ Der folgende Absatz orientiert sich an: Stefan Horlacher: Webster, John: The White Devil. In: Kindlers Literatur Lexikon. Hg. von Heinz Ludwig Arnold. Stuttgart-Weimar: Metzler. 3., völlig neu bearb. Aufl. 2009. Bd. 17. S. $266 f$. 
die Gerichtsszene aus dem englischen Renaissancetheater hervor, da sich die aufgrund ihres Geschlechts als Hure gebrandmarkte Vittoria als ihren männlichen Anklägern verbal deutlich überlegen erweist und die Perfidie einer patriarchalen Logik schonungslos offenlegt. Da diese aber trotz Vittorias Kritik bestehen bleibt, verurteilt sich die Protagonistin letztlich indirekt mit jedem Wort selbst. ${ }^{12}$ Dessen ungeachtet erfolgt auf einer Metaebene die Autodenunziation patriarchaler Mechanismen, da Webster durch die metadramatische Dimension der Gerichtsszene, in der nicht nur die Performativität geschlechtlicher Subjektpositionen deutlich wird, die strukturelle Asymmetrie patriarchaler Machtstrukturen und die diese prägende uneingestandene Differenz zwischen helden- und tugendhaftem Schein einerseits und perfide-intrigierendem Sein andererseits effektvoll anprangert.

Doch selbst wenn sich Vittoria in dieser düsteren Parabel gekonnt der männlichen Diskursmaschinerie bemächtigt und dadurch zur phallischen Frau wird - "my defence, of force, [. . .], / Must personate masculine virtue. To the point". ${ }^{13}$-, kurzfristig agency erlangt und Heuchelei und Misogynie denunziert, so wird sie doch weder belohnt noch exkulpiert und stirbt letztlich "more passive and more victimized than she knows". ${ }^{14}$ Insofern teilt sie einerseits das Schicksal zahlreicher vermeintlich starker Frauen des elisabethanischen und jakobäischen Theaters, bietet andererseits durch ihre schillernde Ambivalenz jedoch eindeutig größere Identifikationsund Empathiemöglichkeiten als eine Tamora, Goneril, Reagan oder Lady Macbeth.

Deutlicher aufgelöst wird diese Ambivalenz erst in Websters 1613/14 verfasster Rachetragödie The Duchess of Malfi, ${ }^{15}$ die einerseits von einer

${ }^{12}$ Begründet wird das Schweigegebot für Frauen u.a. durch die Gleichsetzung von ungezügelter Rede mit ungezügelter Sexualität. Vgl. Theodora A. Jankowski: Women in Power in the Early Modern Drama. Urbana-Chicago: University of Illinois Press 1992. S. 38; Dympna Callaghan: Woman and Gender in Renaissance Tragedy. New York u.a.: Harverster Wheatsheaf 1989. S. $76 f$.

${ }^{13}$ Webster: The White Devil. III.2,138f.

${ }^{14}$ Judith Haber: 'My Body Bestow upon My Women'. The Space of the Feminine in The Duchess of Malfi. In: Renaissance Drama 28 (1997). S. 133-159. Hier: S. 134. Siehe auch ebd.: "The White Devil is a profoundly pessimistic play. It successfully anatomizes but simultaneously cedes to a social and symbolic order that provides women with no place to stand, that offers no mode of existence, other than being 'for-men' or 'like men,' that [. . .] provides no access to a subject-position that does not implicate one in violence toward and violation of the other."

${ }^{15}$ Alle folgenden Zitate sind aus: Five Jacobean Tragedies Middleton/Webster. Hg. von Tom Griffith (General editor), mit Einleitungen versehen von Andrew Hadfield. Ware: Wordsworth 2001. 
dialogischen Struktur der weiblichen Stärke und damit einhergehenden Patriarchatskritik und andererseits von der Dekonstruktion erst dieser starken weiblichen Position und dann der patriarchalen Strukturen selbst geprägt ist. Wie nicht anders zu erwarten, ist die - natürlich von einem Mann gespielte "first fully tragic woman in Renaissance drama" auch hier chancenlos und stirbt sogar schon im vierten Akt. ${ }^{16}$ Nicht nur aus diesem Grund liegt der Hauptkonsens der Webster-Kritik darin, dass man sich eher über die Ambivalenz als über die Gesamtaussage des Theaterstückes einig ist. So schreibt Nancy Lamb Roider:

[T] he Duchess of Malfi has been interpreted in many different ways [. . .]. Some have seen it as a cautionary tale that shows what can happen when women marry without being granted the 'proper' consent, while others argue that it is a feminist story of a strong woman ahead of her time. ${ }^{17}$

Die Frage, wie die eigenwillige und unabhängige Protagonistin zu interpretieren ist, führt dazu, dass Theodora A. Jankowksi von der "ideologically contradictory nature of The Duchess of Malfi" spricht und zwei gegenläufige Diskurse im Stück identifiziert, nämlich "one that validates male family members' rights over the bodies of their female 'property' and one that mandates a woman's free choice as a moral necessity". ${ }^{18}$ Lisa Jardine hingegen sieht "the Duchess's progress toward repentance and death as a masculine wish fulfillment directed at the theater audience" und die Figur der Herzogin selbst "as metamorphosing from a 'strong woman,' the threatening hic mulier, to a comfortably passive figure of female suffering". ${ }^{19}$

\footnotetext{
${ }^{16}$ Frank Whigham: Sexual and Social Mobility in The Duchess of Malfi. In: PMLA 100.1 (1985). S. 167-186. Hier: S. 174.

${ }^{17}$ Nancy Lamb Roider: Arbella Stuart, Catherine of Valois, and The Duchess of Malfi: An Examination of Women, Marriage, and Widowhood in Jacobean England. $<$ http://www.triviumpublishing.com/articles/duchessofmalfi.html $>$. Downloaded 5.2.2005.

${ }^{18}$ Theodora A. Jankowski: Defining/Confining the Duchess. Negotiating the Female Body in John Webster's The Duchess of Malfi. In: Studies in Philology 87.2 (1990). S. 221-245. Hier: S. 232.

${ }^{19}$ Siehe Christy Desmet: 'Neither maid, widow, nor wife'. Rhetoric of the woman controversy in Measure for Measure and the Duchess of Malfi. In: In another country: feminist perspectives on Renaissance drama. Hg. von Dorothea Kehler und Susan Baker. Metuchen. New Jersey-London: Scarecrow Press 1991. S. 71-92. Hier: S. 82; s. Lisa Jardine: The Duchess of Malfi. A case study in the literary representation of women. In: Teaching the Text. Hg. von Susanne Kappeler und Norman Bryson. London-Boston: Routledge \& Kegan Paul 1983. S. 203-217.
} 


\section{Zwischen patriarchaler Dominanz und Entwürfen einer 'weiblichen Ordnung'}

Allein schon aus Platzgründen kann es nicht darum gehen, die oben dargelegten textinhärenten Widersprüche in toto $\mathrm{zu}$ erklären. ${ }^{20}$ Statt dessen soll eine für The Duchess of Malfi spezifische Korrelation zwischen Weiblichkeit, Mutterschaft sowie dem Konzept des Abjekts herausgearbeitet und dabei implizit Luce Irigarays These mitberücksichtigt werden, dass "die ganze Problematik von Raum und Zeit neu betrachtet werden" muss, damit die sexuelle "Differenz gedacht und gelebt werden kann". ${ }^{21}$ Es gilt deshalb zumindest ansatzweise zu fragen, inwiefern The Duchess of Malfi eine Zeitlichkeit wie auch eine Topographie entwirft, die nicht nur die Konstruiertheit von GenderDefinitionen und die Möglichkeit ihrer Inversion verdeutlichen, sondern auch einen Raum sexueller Differenz eröffnen, in dem Weiblichkeit als eigener Ort, und das bedeutet vor allem nicht als Gefäß, als "a box of worm-seed" oder als Körperobjekt für den Mann existiert. ${ }^{22}$ Wenn sich die vorgeschlagene Lesart zumindest teilweise an der französischen 'Differenztheorie' orientiert, die sich selbst gerade nicht als Theorie begreift, ${ }^{23}$ so nicht um deren zweifelsohne inhärente Widersprüche aufzuzeigen, sondern um sie als Kontrastfolie für ein polysemes Theaterstück fruchtbar zu machen. Zudem gilt es zu berücksichtigen, dass die Figur der Herzogin weniger als mimetisches Porträt von Weiblichkeit im frühen 17. Jahrhundert, denn als das Resultat einer am Schnittpunkt der immer auch geschlechtlich codierten Machtstrukturen von Politik, Gesellschaft, Erotik und Familie situierten innovativen Überkreuzung

${ }^{20}$ Für eine detaillierte Analyse des Stücks - allerdings ohne Berücksichtigung des Abjekts und seiner Verbindung zur Mutterschaft - siehe Stefan Horlacher: Zur Frage der Präsenz 'starker Frauen' und der Dekonstruktion des Patriarchats in der Englischen Renaissancetragödie unter besonderer Berücksichtigung von John Websters The Duchess of Malfi. In: Anglia (im Druck). Hieran orientieren sich auch einige der folgenden Ausführungen.

${ }^{21}$ Luce Irigaray: Ethik der sexuellen Differenz. Frankfurt a.M.: Suhrkamp 1991. S. 13.

${ }^{22}$ Webster: The Duchess of Malfi. IV.2,119.

${ }^{23}$ So betont etwa Irigaray, dass es ihr gar nicht um eine traditionelle Theoriebildung gehe, da diese in der bereits bestehenden patriarchalen Logik gefangen bliebe: "Denn von der oder über die Frau zu sprechen kann immer hinaus laufen auf oder verstanden werden als eine Wiederaufnahme des Weiblichen ins Innere einer Logik, die es in der Verdrängung, unter der Zensur, genauer in der Verkennung festhält. Mit anderen Worten, es gilt nicht, eine neue Theorie auszuarbeiten, deren Subjekt oder Objekt die Frau wäre, sondern der theoretischen Maschinerie selbst Einhalt zu gebieten, ihren Anspruch auf Produktion einer viel zu eindeutigen Wahrheit und eines viel zu eindeutigen Sinns zu suspendieren.” Luce Irigaray: Das Geschlecht, das nicht eins ist. Berlin: Merve 1979. S. 80. 
diskursiver Formationen gelesen wird. ${ }^{24}$ Hierbei wird eine prekäre Ambivalenz des Stückes deutlich, die sich einerseits aus der brisanten Stoffwahl, ${ }^{25}$ andererseits aus dem widersprüchlichen Charakter der Protagonistin ergibt, der aus den vermeintlichen Oxymora der weiblichen Herrscherin, ihrer heimlichen Heirat mit einem nicht standesgemäßen Mann, der dynamisch-dynastischen Beziehung zwischen ihrem body politic und ihrem body natural sowie zwischen einem phallisch-stabilen (männlichen) und einem fluid-changierenden (weiblichen) Körperbild resultiert.

Konzentrieren sich traditionelle Lesarten in der Regel auf die Relevanz des patriarchalen setting und der männlich codierten Umgebung für die Interpretation von The Duchess of Malfi, so könnte man tatsächlich die Frage stellen, wo sich ein weiblicher Raum, auch der Subjektivität, auftun sollte angesichts einer sich auf mehreren Ebenen in einer reinen Männerwelt bewegenden Herzogin. Nicht nur die intertextuelle und extratextuell-historische Ebene, sondern auch die Bühnenwelt, in der sich die Herzogin bewegt, ist maskulin konnotiert. Hierbei fällt es den männlichen Charakteren, das heißt ihren Brüdern wie auch dem Spion Bosola, stellvertretend für die Zuschauer zu, sie zu beobachten, zu interpretieren und zu überwachen. Aus diesen Gründen ist die Herzogin bereits zu Beginn des Stücks in ihrem eigenen Palast dazu gezwungen, "schnell einen Schlupfwinkel zu finden, der ihnen entgeht. Sich an einen Ort zurückzuziehen, der ihren Berechnungen, ihren Blicken, ihren Nachforschungen entzogen ist. Ihrem Eindringen. Aber wo?". ${ }^{26}$

Irigarays Zitat beschreibt nicht nur treffend die Situation der Herzogin, sondern zugleich auch die Aufgabe Bosolas, der von Ferdinand, dem Bruder der Herzogin, ganz explizit den Auftrag erhält:

To live i'the court here, and observe the duchess;

To note all the particulars of her haviour,

What suitors do solicit her for marriage,

And whom she best affects. She's a young widow:

I would not have her marry again. ${ }^{27}$

Der “intelligencer" Bosola funktioniert wie die Linse einer Überwachungskamera, aber eben doch nicht ganz: Da nach Freud die Wissbegierde vom Partialtrieb der Schaulust abzweigt, liegt letztere auch dem vermeintlich kalten

${ }^{24}$ Siehe Jankowski: Women in Power. S. 49; Whigham: Sexual and Social Mobility. S. 181.

${ }^{25}$ Siehe Dympna Callaghan: Introduction. In: The Duchess of Malfi. Hg. von Ders. Basingstoke: Macmillan 2000 (New Casebooks). S. 1-24. Hier: S. 8.

${ }^{26}$ Irigaray: Das Geschlecht, das nicht eins ist. S. 11.

${ }^{27}$ Webster: The Duchess of Malfi. I.1,252-256. 
wissenschaftlichen Blick zugrunde, kann das Auge die Spuren seiner körperlichen Herkunft nicht verleugnen. Bosola wird dadurch zur intratextuellen Projektion des Begehrens nicht nur der Brüder der Herzogin, sondern auch eines guten Teils der Zuschauer selbst; eines traditionell männlich codierten Begehrens, alles zu sehen, alles zu wissen und das weibliche 'Objekt' "as its passive, masochistic feminine victim" zu kontrollieren und zu beherrschen. ${ }^{28}$ Notwendig wird dies primär aufgrund der juristischen Identität der Herzogin als Witwe und Herrscherin, die sie zu einer potentiell anormalen und furchteinflößenden Gestalt jenseits patriarchaler Kontrolle macht. ${ }^{29}$ Doch kann die Herzogin weder ihre mehrfachen, vermeintlich paradoxen Identitäten als 'female ruler', 'widowed bride' und 'princely mother' über einen längeren Zeitraum leben, da ihr weder ihre Brüder noch die Gesellschaft die Einnahme derart komplexer Subjektpositionen gestatten, noch kann sie diese in ein kohärentes Verhältnis zueinander bringen. Aufgrund der anhaltend betriebenen Sexualisierung der Herzogin werden ihr aber auch einfache, respektable Subjektpositionen wie beispielsweise diejenige der liebenden Mutter verweigert, da ihre Brüder sie auf die Position der Hure zu reduzieren versuchen. Erschwerend kommt hinzu, dass sich die Herzogin - könnte man ihre Heirat mit dem ihr sozial unterlegenen Haushofmeister Antonio Bologna und ihr Bekenntnis zu ihrer Sexualität vielleicht noch als protoexistentialistisches statement lesen $^{30}$ - wiederholt selbst diskreditiert, beispielsweise wenn sie ihren Brüdern erlaubt, ihre Tugend offen in Frage zu stellen oder wenn sie sich mit einem Diamanten vergleicht, dessen Wert zunimmt, je öfter er von einer Männerhand in die nächste wandert. ${ }^{31}$

Verstärkt wird dieser das Bild der Herzogin prägende widersprüchliche Eindruck durch die Tatsache, dass sie zwar eine aktive Rolle in Bezug auf ihre Heirat mit Antonio einnimmt, ${ }^{32}$ dass sie danach aber die Ehe geheim hält und dadurch erkennen lässt, dass sie keine Möglichkeit sucht, sie in ihr öffentliches Leben als Herrscherin zu integrieren beziehungsweise ihre 'beiden Körper' in ein sozial akzeptiertes Verhältnis zueinander zu bringen. Statt dessen unterschätzt sie die Auswirkungen ihres privaten auf ihr öffentliches Leben, ihres body natural auf ihren body politic, ${ }^{33}$ und betont, indem sie gleich mehrfach schwanger wird, unfreiwillig ihre biologische Weiblichkeit, wodurch

${ }^{28}$ Toril Moi: Sexual/Textual Politics. Feminist Literary Theory. London-New York: Methuen. 9. Aufl. 1990. S. 180.

${ }^{29}$ Siehe Barbara J. Todd: The Remarrying Widow: A Stereotype Reconsidered. In: Women in English Society 1500-1800. Hg. von Mary Prior. London-New York: Methuen 1985. S. 54-92; siehe auch Jankowski: Women in Power. S. 35f.

${ }^{30}$ Webster: The Duchess of Malfi. III.2,11.

${ }^{31}$ Ebd. I.1,298ff.

${ }^{32}$ Ebd. I.1,438ff.

${ }^{33}$ Siehe Jankowski: Defining/Confining the Duchess. S. 223. 
sie in der Öffentlichkeit stärker als Frau und potentielle Hure denn als Herrscherin wahrgenommen wird. So nimmt auch ihre ursprünglich befreiende und unkonventionelle Heirat zunehmend die ironische Wendung eines geheimen, immer klaustrophobischeren Rückzugs an: Erst muss die Herzogin ihr Privatleben und ihre drei Schwangerschaften verbergen, dann wird sie von ihren Brüdern in ihrem eigenen Palast gefangen gehalten, letztlich wird sie isoliert, mit Wahnsinnigen umgeben, gefoltert und getötet. Gerade die vermeintliche Macht und Unabhängigkeit der Herrscherin und Witwe erweisen sich schon bald als Illusion, denn als sie von ihrer Macht Gebrauch machen möchte, beginnt ihr irreversibles Scheitern, wodurch die Probleme nur noch deutlicher werden, die sich beim Entwurf einer weiblichen Subjektivität wie auch weiblicher Raum- und Zeitstrukturen ergeben.

Lassen es die bisherigen Darlegungen eher fraglich erscheinen, ob es dem Stück gelingt, patriarchale Machtstrukturen zu hinterfragen, zu dezentrieren und letztlich auch zu transzendieren, so haben Kritiker argumentiert, dass bereits die disruptive Form des Stücks selbst die patriarchale Ordnung subvertiere. ${ }^{34}$ Von besonderer Bedeutung ist hierbei, dass Websters Stück die Dimension der Zeit explizit und direkt an die Schwangerschaften der Herzogin bindet - "Since you last saw her / She hath had two children more"35 und die Namensgeberin des Stücks bereits im vierten Akt stirbt, wodurch dem fünften Akt, einem ebenso seltsamen wie interessanten Appendix, eine besondere Bedeutung zukommt. Mit Irigaray könnte man argumentieren, dass The Duchess of Malfi durch seine potentiell defiziente Form, vor allem aber durch seinen Inhalt die Tatsache problematisiert, dass die Illusion männlicher Reinheit, Ganzheit und Einheit von einer gewaltsamen Aneignung und Besitzergreifung des weiblichen Körpers und einer damit verbundenen Negation weiblicher Subjektivität abhängt. Wenn die Frau nicht nur das Andere, sondern sehr viel spezifischer das Andere des Mannes ist, nämlich sein Negativ oder Spiegelbild, dann imitiert oder mimt die Herzogin als repetition with a difference einerseits den patriarchalen Diskurs - sie ist selbst Herrscherin und verwendet wiederholt kriegerische Topoi -, andererseits subvertiert sie die nach Hélène Cixous von Kastrationsangst geprägte und aus Verlustangst nur einen kontrollierten (Waren-)Austausch zulassende männliche Ökonomie des Selben (realm of the proper), ${ }^{36}$ indem sie versucht, ihren Körper fremder

\footnotetext{
${ }^{34}$ Siehe auch Callaghan: Introduction. S. 1f.; Haber: 'My Body Bestow upon My Women'. S. 135; R.S. White: The Moral Design of The Duchess of Malfi. In: The Duchess of Malfi. Hg. von Dympna Callaghan. Basingstoke: Macmillan 2000 (New Casebooks). S. 201-216. Hier: S. 203.

${ }^{35}$ Webster: The Duchess of Malfi. III.1,6f.

${ }^{36}$ Siehe hierzu die kritische Darstellung bei Lena Lindhoff: Einführung in die feministische Literaturtheorie. Stuttgart: Metzler. 2. Aufl. 2003. S. 114f.
} 
Kontrolle zu entreißen und sich zu ihrem sexuellen Begehren zu bekennen. Sie entzieht sich somit einer von Ferdinand und dem Kardinal verkörperten patriarchalen Logik, in der sie nie mehr als passives Tauschobjekt zwischen Männern sein kann, denn, so Irigaray:

[D]ie Frau ist traditioneller Weise Gebrauchswert für den Mann, Tauschwert zwischen den Männern. Ware also. Was sie Hüterin der Materie sein läßt, deren Preis nach dem Maß ihrer Arbeit und ihres Bedürfnis-Wunsches von den 'Subjekten' festgesetzt wird: Arbeitern, Händlern, Konsumenten. Die Frauen werden durch ihre Väter, Ehemänner, Kuppler phallisch markiert. Und diese Abstempelung entscheidet über ihren Wert im sexuellen Handel. ${ }^{37}$

Doch die Herzogin lässt sich nicht vereinnahmen, sondern schafft einen zirkulären weiblichen Raum beziehungsweise eine Sphäre, die frei von feindlicher, und das heißt in der Regel männlicher Invasion ist: Dem karikaturesk-phallischen Dolch ihres Bruders Ferdinand setzt sie mit "All discord without this circumference / is only to be pitied, and not feared" einen geschützten Bereich entgegen, ${ }^{38}$ den Judith Haber als aus ihren Armen bestehend, in denen sie ihren Geliebten hält, ihrem Ring, der Figurenkonstellation mit Antonio und Cariola in ihrem Schlafzimmer, aber auch als "the circular form of her sexuality and of her pregnancies" identifiziert. ${ }^{39}$ Innerhalb dieser circumference setzt zwischen der Herzogin, Antonio und Cariola ein verbales spielerischerotisches Reizen ein, das aber nicht auf die sexuelle Erregung Antonios, sondern auf die der Herzogin gerichtet ist ${ }^{40}$ - wodurch diese von der patriarchalen Ordnung einmal mehr als Bedrohung erfahren wird, denn

[w]ie kann dieses Tauschobjekt ein Recht auf Lust beanspruchen, ohne aus dem etablierten Handel auszusteigen? [. . .] Wie könnte die Materie sich selbst genießen, ohne beim Konsumenten Angst vor dem Verschwinden seines Nährbodens zu provozieren ${ }^{41}$

Kritikerinnen wie Jankowski und Haber sprechen sogar von einem anhand der Herzogin exemplifizierten parler femme, und in der Tat kehrt die Herzogin zumindest vorübergehend die patriarchale Ordnung aktiv um und überschreibt sie kreativ. ${ }^{42}$ Dabei ersetzt sie die männliche jedoch nicht durch eine weibliche Ordnung - bliebe sie dabei doch in der gleichen Logik gefangen -, sondern führt ein spielerisch-parodistisches Element und somit

\footnotetext{
${ }^{37}$ Irigaray: Das Geschlecht, das nicht eins ist. S. 31.

${ }^{38}$ Webster: The Duchess of Malfi, I.1,328ff.; ebd. I.1.,465f.

${ }^{39}$ Haber: 'My Body Bestow upon My Women'. S. 138.

${ }^{40}$ Ebd. S. $138 f$.

${ }^{41}$ Irigaray: Das Geschlecht, das nicht eins ist. S. 31.

${ }^{42}$ Webster: The Duchess of Malfi. III.2,3-6.
} 
eine Metaebene ein. ${ }^{43}$ Ist in einem ersten Schritt während ihrer Schwangerschaft nicht die Herzogin eingeschlossen, sondern sind es die Offiziere, die ihre Quartiere nicht verlassen dürfen, so wird in einem zweiten Schritt diese Umkehrung überschritten, wenn sie Antonios Sexualität als weibliche reimaginiert. Indem sie ihre Brüder als "gossips", also Klatschbasen, bezeichnet, ${ }^{44}$ bringt sie nicht sich, sondern ihren Ehemann indirekt in die Position der schwangeren Frau und ihre Brüder in die der Freundinnen, die traditionell eingeladen wurden, um der Schwangeren am Wochenbett beizustehen und unter Ausschluss der Männer eine kollektiv-weibliche Sphäre zu schaffen. ${ }^{45}$

Besteht nach Irigaray die traditionell männliche Konzeption von Integrität darin, allein, funktionsfähig und 'ganz' zu sein, und ist gemäß dieser Logik penetriert zu werden mit einem absoluten Machtverlust gleichzusetzen, dann besteht Integrität für die Herzogin nicht einfach darin, eine männliche Subjektposition einzunehmen, das heißt, unpenetrierbar zu werden oder ihre Sexualität zu verleugnen, sondern darin, aktiv zu wählen, "who may enter her". Die Herzogin wird als eine Frau porträtiert, die ihre eigene Sexualität kontrolliert, nicht jedoch indem sie ihren Körper verweigert oder sich in patriarchaler Tradition einfach nimmt, was sie begehrt, sondern indem sie eine Beziehung zu Antonio einfordert, in der beider Sexualität Genüge getan, in der sie respektiert und auch erfüllt wird. ${ }^{46}$ Wenn sich Antonio beschwert: "These words should be mine" und die Herzogin feststellt: "You speak in me this", ${ }^{47}$ so spielt sie auf die Logik der Penetration an, invertiert die Subjektpositionen und macht dadurch auch deren Konstruiertheit sichtbar. Und wenn sie Antonio mitteilt: "I entered you into my heart / Before you would vouchsafe to call for the keys", 48 dann steht dies für eine spezifisch weibliche Handlungsmächtigkeit oder agency, hat sie ihm (und somit einer potentiell patriarchalen Sprache und Logik) doch bereits zuvor das Wort entzogen - "I'll stopp your mouth. [kisses him]"49 -, nicht aber, um ihn mundtot zu machen oder zum Objekt ihrer Lust $\mathrm{zu}$ degradieren, sondern um einen gleichberechtigten Dialog zu ermöglichen.

\footnotetext{
${ }^{43}$ Haber: 'My Body Bestow upon My Women'. S. 144.

${ }^{44}$ Eine weitere Bedeutung von to gossip ist "to speak as a woman"; siehe ebd. S. 142f. u. 154, Anm. 27.

${ }^{45}$ Könnte man versucht sein, diese Sphäre als kurzfristigen, in die patriarchale Ordnung eingebetteten karnevalesken Ausnahmezustand und somit als indirekte Bestärkung des Patriarchats zu lesen, so falsifiziert Websters Stück diese Interpretation, indem es diesen Zustand zum mehrere Jahre andauernden Realzustand macht, der nachhaltig die patriarchale Ordnung genauso wie ihre Werte suspendiert.

${ }^{46}$ Siehe Jankowski: Defining/Confining the Duchess. S. 230f. u. 235.

${ }^{47}$ Webster: The Duchess of Malfi. I.1,467; ebd. I.1,490.

${ }^{48}$ Ebd. III.2,62f.

${ }^{49}$ Ebd. III.2,22.
} 
Dies führt zu einer von Cixous als "unendliche Zirkulation des Begehrens" beschriebenen Beziehungsstruktur, die es erlaubt, "von 'Liebe' zu sprechen, ohne mit diesem Wort sein Gegenteil, tödliche Aggression gegen den Anderen, zu verdecken", ${ }^{50}$ und die eine Anerkennung voraussetzt,

die nicht Kampf um Vorherrschaft wäre, sondern eine wechselseitige Anerkennung zweier unterschiedlicher Subjekte, die eine Erkenntnis des Anderen als Anderen anstreben würde, ohne sich von seinem Anderssein bedroht zu fühlen. ${ }^{51}$

Die hierdurch dokumentierte patriarchatsfremde Logik des realm of the gift als eine Ökonomie des freizügigen Gebens stellt eine akute Bedrohung für die traditionellen Machtstrukturen dar und kann nur an einem Ort existieren, an dem die Gesetze des Patriarchats suspendiert sind. Websters Stück spricht seiner Protagonistin diesen Raum immerhin für drei Akte oder drei Schwangerschaften $\mathrm{zu}$ - und wie letztere eindrucksvoll beweisen, ist dieser weibliche Raum nicht fiktiv oder karnevalesk, sondern transgressiver als jede Fiktion und funktioniert binnenfiktional als Alternative zum Patriarchat und der dieses kennzeichnenden Triebökonomie. Was immer von letzterer in die circumference der Herzogin durchdringt, verpufft wirkungslos: So kommentiert die Herzogin Bosolas Invektiven gegen Kosmetik wie auch ältere Frauen indirekt dadurch, dass sie sich auf positive Art und Weise mit dem körperlichen Prozess des Älterwerdens auseinandersetzt, ${ }^{52}$ gleichzeitig spielerisch Ferdinands Spiegellogik ${ }^{53}$ imitiert und die Dimension der Zeitlich- und Veränderlichkeit nicht etwa ausblendet, sondern bejaht. ${ }^{54} \mathrm{Da}$ sie darüber hinaus ihre Fähigkeit zur Inversion gerade nicht spiegelbildlich zu Ferdinand, das heißt etwa zur kalkulierten Machterhaltung eines weiblichen Regimes einsetzt, leistet sie deutlich mehr als eine bloße Positionsumkehrung:

[S]he paradoxically confirms the validity of the image she creates. For as she sits, in classically 'feminine' fashion, before her looking glass, she is, in effect, stepping through that looking glass to remake the world in her own image (by troping on the patriarchal constructions out of which that image is made), all the while selfconsciously acknowledging (and this is part of her project) that she is engaging in 'sportive action;' rather than 'action indeed'. ${ }^{55}$

Die Doppelung von "sportive action" und "action indeed" die von Bosola vehement kritisierte, weil vom männlichen Blick nicht erkennbare

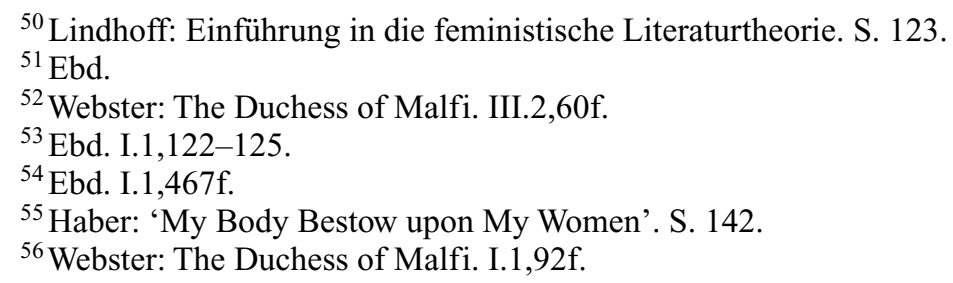


Doppelstruktur zwischen männlich-diskursiver Realität und weiblicher Mimikry, zwischen patriarchalen Überwachungsmechanismen und einer ihnen nicht fassbaren weiblichen Sphäre, zwischen dem wissenschaftlich-voyeuristischen Blick zugänglicher Oberfläche und als Täuschung empfundenem Make-up. ${ }^{57}$ Patriarchale Linearität, Sprache und Logik weichen dem Zyklus der Schwangerschaften und dem gossip beziehungsweise einem "playful, women-centered chatter - a kind of Renaissance parler-femme", 58 mit dem Klassen-, Macht- und Penetrationsdiskurse aufgenommen und in einem weiblichen Raum invertiert und transzendiert werden. Zwar wird diese diskursiv-kreative Verarbeitung von Tatsachen zu einer der Herzogin eigenen Realität vom Text an die Modelle des 'gossip', der 'old wives tales' und der 'contemporary romances' angelehnt, doch stellt diese Anbindung keine Denunziation der weiblichen Sphäre als rein fiktionaler und deshalb in der Lebenswirklichkeit nicht wirkmächtiger Raum dar, hat sich doch die von der Herzogin geschaffene Realität für einige Jahre als funktionsfähig erwiesen. Könnte man argumentieren, dass die Herzogin ihren Lebensentwurf nicht einer vermeintlich rationalen spekularen Logik des Selben, sondern den gerade thematisierten stärker semiotischen literarischen Formen verdankt, so könnten die bereits angesprochenen politisch unklugen Inkonsistenzen in ihrem Verhalten als ein Unterlaufen einer männlich-spekularen Logik interpretiert werden, der sie eine weibliche 'Logik' oder Sprache entgegensetzt, die sich “durch labile Signifikationsverhältnisse, metonymische Verfahren und differente Sinnbildungsprozesse auszeichnet". ${ }^{59}$ Und so sehr das Festhalten der Herzogin an einer semiotischen Sprache wie auch ebensolchen literarischen Formen ihren Untergang beschleunigen mag, so erreicht sie textstrategisch doch einen Freiraum, in dem sich zumindest die Andeutung einer Frau konstituieren kann, die mehr und anders ist als das positive oder negative Spiegelbild des Mannes und die ihre 'Nicht-Fixierbarkeit' einem ambivalenten Status zwischen Objekt und enigmatischem Subjekt verdankt.

Der Preis, den das Theaterstück für die innovative Subjektkonstruktion seiner Protagonistin zahlt, liegt darin, dass es - auch wenn eine sich an der Biologie orientierende Konzeption von Weiblichkeit nicht notwendigerweise essentialistisch ist - zumindest potentiell Gefahr läuft, die der theoretisch problematischen Konzeption eines parler femme beziehungsweise einer écriture féminine inhärenten essentialistischen Einsprengsel, aber auch Stereotype von

\footnotetext{
${ }^{57}$ Siehe Jankowski: Defining/Confining the Duchess. S. $237 \mathrm{f}$.

${ }^{58}$ Haber: 'My Body Bestow upon My Women'. S. 144.

${ }^{59}$ Gabriele Rippl: Feministische Literaturwissenschaft. In: Einführung in die Literaturwissenschaft. Hg. von Miltos Pechlivanos u.a. Stuttgart-Weimar: Metzler 1995. S. 230-240. Hier: S. 235; siehe auch Irigaray: Das Geschlecht, das nicht eins ist. S. 28.
} 
Weiblichkeit fortzuschreiben. Es bleibt nämlich festzuhalten, dass - obwohl während der Ehe mit Antonio konventionelle Geschlechterrollen transzendiert werden und weibliche agency privilegiert wird - es in letzter Konsequenz die Schwangerschaften der Herzogin sind, die ihren Niedergang bedingen. Trotzdem wird die Herzogin nicht auf ihre Biologie reduziert: Zwar definiert der Text Weiblichkeit und weibliche Räume über Schwangerschaft und schreibt die Herzogin in ein altes Naturparadigma ${ }^{60}$ ein -“A woman as mother would be [...] a strange fold that changes culture into nature, the speaking into biology". 61 "In der Gestalt des Vaters personifiziert sich die Macht der Gesellschaft, in derjenigen der Mutter die der Natur"62 -, doch wird das Motiv der Schwangerschaft nicht etwa genutzt, um weibliche Sexualität zu limitieren oder ganz zu beenden und die sexuell aktive Frau auf ihre Mutter- beziehungsweise Dienstleistungsfunktion zu reduzieren. ${ }^{63}$ Vielmehr wird dieses Motiv strategisch einer pervertierten patriarchalen Ordnung entgegengesetzt und entlarvt deren vermeintliche Werte und Ängste als sich als 'Sorge um die Reinheit der Blutlinie' artikulierendes inzestuöses Begehren, als Angst vor Privilegienverlust und sozialem Abstieg sowie als Fetischisierung einer wirtschaftlichen Logik, nach der die Herzogin einschließlich ihrer biologischen Fähigkeit, Kinder zu gebären, lediglich ein geschlechtsspezifischer Körper ist, dessen Gebrauch, Konsum und Zirkulation "die Organisation und die Reproduktion der Gesellschaftsordnung" sichert, ohne dass sie an dieser jemals als Subjekt

60 "Es gibt nichts, was einander ähnlicher wäre als das abendländische Ideal der Mütterlichkeit und die Affenliebe. Auf keinem anderen Gebiet beruft sich der 'Kulturmensch' so sehr wie hier auf die Verwandtschaft mit dem Tier. Während er sich überall sonst gerade durch die Unterscheidung vom Tier auszeichnet, ja in ihr die Definition des 'Menschen' sieht, macht er für die Bande, die Mutter und Kind verbinden, das Gesetz des 'Instinkts', der 'Natur', der 'Reflexe' geltend." Christina von Braun: Nicht Ich. Logik, Lüge, Libido. Frankfurt a.M.: Neue Kritik. 3. Aufl. 1990. S. 210.

${ }^{61}$ Julia Kristeva: Stabat Mater. In: The Kristeva Reader. Hg. von Toril Moi. New York: Columbia UP 1986. S. 160-186. Hier: S. 182. Luce Irigaray: Speculum. Spiegel des anderen Geschlechts. Frankfurt a.M.: Suhrkamp 1980. S. 17 u. 66 warnt vor der Identifizierung des Weiblichen mit dem Mütterlichen. Siehe auch Irigaray: Das Geschlecht, das nicht eins ist. S. 63 u. 90; Adrienne Rich: Of Woman Born. Motherhood as Experience and Institution. New York: Bantam 1976; Mutterschaft. Mythos und Zukunft. Hg. von Gerd-Klaus Kaltenbrunner. München: Taschenbuch-Magazin Initiative 1987.

${ }^{62}$ Suchsland: Julia Kristeva. S. 129; vgl. ebd. S. 147.

63 "[T] he association of pregnancy here with sexual pleasure and bodily excess on the one hand and with female sociality on the other make it potentially threatening to a patriarchal order in ways that have been lost or muted in its current desexualized and medicalized form.” Haber: 'My Body Bestow upon My Women'. S. 150. 
teilhätte. ${ }^{64}$ Nach Irigaray ist dies eine Gesellschaftsordnung, in der erst über das Objekt Frau die Beziehung(en) zwischen Männern ermöglicht und Gesellschaft initiiert wird; eine Gesellschaftsordnung folglich, in der die Umbildung des Frauenkörpers in Gebrauchs- und Tauschwert die symbolische Ordnung inauguriert. $^{65}$

Wenn aber die Herzogin durch ihr Handeln eine eigene weibliche Identität und Subjektposition erreicht hat, und wenn der Boden, auf dem Ferdinands Ideologie des reinen Blutes und seiner eigenen reinen Existenz errichtet ist, der einer spekularen Weiblichkeit ist, die er nur als leeres und passives Gefäß oder aber hochglänzenden Spiegel imaginieren kann, wenn das Blut der Herzogin sein Blut ist, wenn ihre weibliche Keuschheit seine männliche Ehre widerspiegelt und wenn er als sich im weiblichen Spiegel betrachtendes männliches Subjekt nur so lange existieren kann, wie der Herzogin eine eigene Subjektivität verwehrt bleibt, dann wird der Tod der Herzogin zur absoluten Notwendigkeit für die Aufrechterhaltung patriarchaler Identität in ihrer reinsten Form. ${ }^{66}$ Folglich muss der von der Herzogin geschaffene weibliche Raum angegriffen und von der herrschenden Ordnung penetriert werden. ${ }^{67}$ Eine überzeugende Gegenwehr billigt Webster der Herzogin nicht zu, denn als Ferdinand beschließt zu handeln, wird ihr Anwesen wie auch die gesamte Bühne von patriarchalen Strukturen in Besitz genommen und in ein Gefängnis verwandelt. Allerdings greifen auch hier sympathielenkende Mechanismen, denn Ferdinand wird als Hauptvertreter des Patriarchats sogar noch über die bereits genannten Beispiele hinaus eindeutig negativ charakterisiert und seine Logik des Selben als die einer gewaltvollen Penetration und somit als eine Logik der Vergewaltigung denunziert. ${ }^{68}$

\section{Männliche Transparenz, weibliches Echo und die Wiederkehr des Abjekts}

"Dispose my breath how please you; but my body / Bestow upon my women, will you". ${ }^{69}$ Indem die Herzogin Ferdinand ihren Leichnam verweigert, entzieht sie sich auch nach ihrem Tod patriarchalen Strukturen und schließt an den bereits von ihr geschaffenen weiblichen Raum an. Erst im Moment ihres Todes verbindet sie ihre Rollen als Mutter und Herzogin, ihre private und öffentliche Existenz, ihren body natural und ihren body politic, da sie sich sowohl explizit um ihre Kinder kümmert als auch auf ihrer politischen Stellung

\footnotetext{
${ }^{64}$ Siehe Irigaray: Das Geschlecht, das nicht eins ist. S. 86f.

${ }^{65}$ Siehe ebd. S. $191 \mathrm{ff}$.

${ }^{66}$ Siehe Irigaray: Speculum. S. 56ff.

${ }^{67}$ Webster: The Duchess of Malfi. I.1,328ff.

${ }^{68}$ Siehe Haber: 'My Body Bestow upon My Women'. S. 140.

${ }^{69}$ Webster: The Duchess of Malfi. IV.2,223f.
} 
besteht. ${ }^{70}$ Diese Betonung ihrer politischen Identität schreibt die Herzogin zumindest zu einem gewissen Grad in die patriarchale Ordnung ein und kann doppelt ironisch gelesen werden, denn einerseits besinnt sie sich ausgerechnet dann auf ihre politische Identität, als diese de facto nicht mehr existiert oder zur Fiktion geworden ist, ${ }^{71}$ andererseits geschieht diese scheinbare politische Validierung in einem Kontext, der primär ihre Identität als Ehefrau und Mutter betont. ${ }^{72}$ Hat die Kritik argumentiert, die Herzogin müsse sterben, weil ihr während des gesamten Stücks keine adäquate Subjektposition angeboten werde, so entzieht sie sich sogar noch im Tod der Appellationsstruktur der symbolischen Ordnung wie auch den Rastern patriarchaler Benennung. Einerseits bleibt sie anonym, da das Publikum zu keiner Zeit ihren Namen erfährt, andererseits führt nicht einmal ihr Tod $\mathrm{zu}$ einem klaren Ende im Sinne einer closure: "[S]he gives us, typically, not one but a series of 'dying lines" ". ${ }^{73}$ Zudem prägt die Herzogin den fünften Akt so nachhaltig durch ihre Absenz, dass dieser völlig zu Recht als "the disembodied, confused echo of the Duchess's death" bezeichnet werden kann. ${ }^{74}$ Wenn Haber argumentiert, Websters Stück versuche "to construct a subjectivity that is specifically female, to reimagine speech, sexuality, and space - most particularly, the space of the female body - in 'feminine' terms", wobei Websters Herausforderung an die "erotics of patriarchy and the structure of conventional tragedy to the desire for a self-defining, self-defeating moment of phallic orgasm and death - [. . .] in terms of reproductive sexuality" gefasst sei, ${ }^{75}$ so geht das Stück im letzten Akt sogar noch klar über Habers Thesen hinaus.

Erst mit dem Tod der Herzogin wird deutlich, dass auch für die männlichen Protagonisten die der männlichen Libido wie auch der klassischen Tragödie vermeintlich inhärenten und von Ferdinand eindrücklich symbolisierten Phantasien von Ganzheit, Geschlossenheit und Reinheit (nicht nur der Blutlinie) nicht lebbar sind. "Purity is on the edge of evil", schreibt Ursula LeGuin, ${ }^{76}$

${ }^{70}$ Ebd. IV.2,197ff.; vgl. ebd. IV.2,118, 129, 137. Problematisch bleibt allerdings die Tatsache, dass sich die Herzogin auch in alte Frauenbilder wie diejenigen der Märtyrerin und der liebenden Mutter einschreibt (Webster: The Duchess of Malfi, IV.1,73f.). Siehe Jankowski: Defining/Confining the Duchess. S. 242f; Jardine: The Duchess of Malfi. S. 207f. u. 215 sowie Desmet: 'Neither maid, widow, nor wife'. S. 84f.

${ }^{71}$ Webster: The Duchess of Malfi. III.4,30-33.

${ }^{72}$ Siehe Whigham: Sexual and Social Mobility. S. 174.

${ }^{73}$ Haber: 'My Body Bestow upon My Women'. S. 147.

${ }^{74}$ Ebd.

${ }^{75}$ Ebd. S. 135.

${ }^{76}$ Ursula Le Guinn: Always Coming Home. Zit. nach Keith Sagar: Open Self and Open Poem. The Stages of D.H. Lawrence's Poetic Quest. In: The D.H. Lawrence Review 24.1 (1992). S. 43-56. Hier: S. 49. 
und ein seines Spiegels beraubtes narzisstisches Ego geht letztlich an seiner Einmaligkeit wie auch an der Transparenz der von ihm geschaffenen Ordnung zugrunde. Bringt der Tod der Herzogin eine patriarchale Ordnung hervor, in der Frauen, wenn überhaupt, dann ausschließlich als Funktion und in Abhängigkeit von Männern existieren, in der ihnen, wie der Text am Charakter von Julia verdeutlicht, jede Spur eines auch nur andeutungsweise refraktären Subjektstatus verweigert wird, so erweist sich diese Ordnung gerade aufgrund ihrer vermeintlichen Reinheit ${ }^{77}$ als nicht lebensfähig und wird in ihrer irrignarzisstischen Logik der Selbstbespiegelung explizit bloßgestellt. Das rein patriarchale, alles Weibliche und potentiell Nicht-Transparente ausschließende System bewirkt in einer "Pathologie des Inzest[s], verstanden in einem weiten und metaphorischen Sinn", seinen eigenen Untergang: "Wer vom Gleichen lebt, kommt durch das Gleiche um". ${ }^{78}$

Websters Stück ist hierbei paradigmatisch und seiner Zeit voraus, gilt der von ihm porträtierte Mechanismus doch sowohl in medizinischen, psychologischen als auch in sozialen, informationsverarbeitenden und technologischen Systemen. Gerade weil der soziale Körper im fünften Akt eine rein patriarchale Homogenität annimmt, verurteilt er sich zum Verschwinden:

Das Gespenst des Gleichen hat wieder zugeschlagen. In dem ganzen Zwang zur Ähnlichkeit, in der Ausmerzung der Differenz, in der Annäherung der Dinge an ihr eigenes Bild [. . .] liegt die Drohung einer inzestuösen Virulenz, einer teuflischen Andersheit, die diese ach so schöne Maschinerie zerstören wird. ${ }^{79}$

Was Jean Baudrillard hier spekulativ-medientheoretisch $\mathrm{zu}$ fassen sucht, beschreibt Julia Kristevas psychoanalytisch orientierte Theoriebildung folgendermaßen:

Jedem System, das die Heterogenität von Subjekt und Sinngebungsprozeß und damit die prinzipielle Unvollkommenheit des Symbolischen leugnet, liegt dasselbe Muster zugrunde. Jedes in sich geschlossene symbolische System, das sich als solches behaupten will, stellt einen Versuch dar, die beiden Momente von Subjekt und Sinn auseinanderzureißen und läuft Gefahr, in die Falle paranoider Projektionen zu tappen. ${ }^{80}$

Hieraus folgt die These, dass sich das von Baudrillard beschriebene Phänomen in The Duchess of Malfi auf der psychosozialen Ebene der symbolischen Ordnung als Einsprengsel der semiotischen, mütterlich konnotierten chora in

${ }^{77} \mathrm{Zu}$ Reinheit im hier gemeinten Sinn siehe Jean Baudrillard: Die Transparenz des Bösen. Ein Essay über extreme Phänomene. Berlin: Merve 1992. S. 72f.

${ }^{78}$ Ebd. S. 75.

${ }^{79}$ Ebd. S. $75 f$.

${ }^{80}$ Suchsland: Julia Kristeva. S. 157. 
Form des Abjekts manifestiert, das heißt in Form eines unfassbaren "Etwas, das sich gleichzeitig mit der Entwicklung des Ichs herausbildet" und "dessen dunkler Schatten" ist:

Es ist noch nicht Objekt, aber schon Nicht-Ich, d.h. es hat noch nicht die klaren Konturen eines vom Ich Unterschiedenen. Es ist die mütterliche chora, von der das Ich sich abzugrenzen sucht. [. . .] Mütterlich ist die chora, insofern sie körperlicher Urspung ist und somit das dem Symbolischen, der Gesellschaft, dem Ich Heterogene. Sie ist weiblich als das dem Phallus gegenübertretende Andere. ${ }^{81}$

Analog zu Baudrillard fallen Reinheit und Tod auch in Kristevas theoretischer Konzeption insofern zusammen, als Kristeva Lacans symbolische Ordnung um das in ihr wirkende, durch eine thetische Phase getrennte Semiotische ergänzt und in der sich vor allem in der Kunst manifestierenden Wiederaufnahme einer vorsignifikanten, polymorphen und präödipalen semiotischen chora nicht nur den Aggressor, sondern vor allem auch die Bedingung selbst des Symbolischen sieht. ${ }^{82}$ Indem sich die Herzogin posthum patriarchalen Strukturen entzieht, bewirkt ihre Abwesenheit, dass die patriarchale Maschinerie - in Anlehnung an Baudrillard könnte man von nahezu absoluter Transparenz, in Anlehnung an Jean-Paul Sartre und D.H. Lawrence von fehlender Viskosität sprechen ${ }^{83}$ - heißläuft und implodiert, sind doch patriarchale Strukturen in ihrer vermeintlich reinsten Form bei Webster für Paranoia

${ }^{81}$ Ebd. S. 123 u. 111. Zum Konzept des Abjekts siehe Julia Kristeva: Powers of Horror. New York: Columbia University Press 1982; Anna Smith: Julia Kristeva. Readings of Exile and Estrangement. London: Macmillan 1996.

${ }^{82}$ Hierbei wird "das Symbolische, die Differenz, Struktur und Stabilität des Ichs" vom Phallus repräsentiert, doch muss die "symbolische (gesellschaftliche) Ordnung [. . . ] ihre Stabilität stets aufs Neue gegen das behaupten, woraus sie hervorgegangen ist, und was sie niemals ganz hinter sich zu lassen vermag: die semiotische chora." Diese wiederum erscheint im Rückblick "als dunkler Ursprung, als Chaos, das sich jedem Zugriff entzieht, als Schmelztiegel des Sinns". Suchsland: Julia Kristeva. S. 111; siehe auch ebd. S. 96.

${ }^{83}$ Siehe Irigaray: Das Geschlecht, das nicht eins ist. S. 110-124. In La Nausée wird Sartres Antoine Roquentin zunehmend vom süßlich-stickigen Geruch des Ekels und der klebrig-schlammigen und feucht-nassen, eine klare Grenze negierenden Unterseite eines Kieselsteins in eine Männlichkeit und Weiblichkeit verwischende und im Selbstmord endende psychische Dekompensation getrieben. Wenn Roquentin somit eine schwere Viskosität die Sinnlosigkeit der eigenen Existenz enthüllt, so steht Viskosität zum Beispiel in Lawrences The Virgin and the Gipsy für ein übergreifendes mütterliches Prinzip, das die Abgrenzung und Individuation des Subjekts zu verhindern sucht und implizit Sartres vielsagenden Vergleich von Weiblichkeit und Honig evoziert. 
und Wahnsinn anfällig, ja treiben sie sogar hervor in Analogie zu Baudrillards medientheoretischen Überlegungen:

Alle integrierten und überintegrierten Systeme, die technischen Systeme, das soziale System, das Denken selbst in der künstlichen Intelligenz und ihren Ableitungen tendieren zu dieser Grenze der Immunschwäche. Da sie alle äußere Aggression abzuschaffen bestrebt sind, sondern sie ihre eigene innere Virulenz, ihr Umschlagen ins Bösartige ab. Bei einem bestimmten Sättigungsgrad übernehmen sie selbst, ohne es zu wollen, diese Funktion der Umkehrung und Zerrüttung und gehen ihrer eigenen Zerstörung entgegen. Ihre Transparenz bedroht sie. ${ }^{84}$

Während zu Lebzeiten der Herzogin die männlichen Phantasien von Ganzheit und Größe auf der Inhaltsebene des Stücks phantasmatisch waren und gerade deshalb lebbar blieben, belegt der fünfte Akt und die darin erfolgende Selbstzerstörung der patriarchalen Maschinerie, dass eine in ihrer reinsten Form ebenso phallozentrische wie tödliche symbolische Ordnung ${ }^{85}$ ohne das Semiotische nicht existieren kann, welches sich in Form des Abjekts manifestiert, Ursprung und Gegenspieler des Symbolischen ist, dessen Regeln subvertiert und das Subjekt spüren lässt, dass es eine provisorische und fragile Struktur ist. Dem entspricht, dass sich das Abjekt nicht nur in körperlichen Verfallsphänomenen zeigt, "sondern auch in allen Verhaltensweisen, die die soziosymbolische Ordnung unterlaufen, ohne sie offen anzugreifen: in Heuchelei, Korruption, Perversion" $" 86$ :

Any crime, because it draws attention to the fragility of the law, is abject, but premeditated crime, cunning murder, hypocritical revenge are even more so because they heighten the display of such fragility. [. . .] Abjection is immoral, sinister, scheming, and shady: a terror that dissembles, a hatred that smiles, [. . .] a friend who stabs you. ${ }^{87}$

Genau diese Verhaltensweisen sind es, die zusammen mit den bereits thematisierten paranoiden Projektionen die von Ferdinand und dem Kardinal errichtete symbolische Ordnung kennzeichnen. Wie die Herzogin früh antizipiert, ${ }^{88}$ wendet sich der 'weibliche' Wahnsinn, ${ }^{89}$ mit dem sie während ihrer Gefangenschaft konfrontiert wird, gegen Ferdinand, dessen Glaube an Reinheit und

\footnotetext{
${ }^{84}$ Baudrillard: Die Transparenz des Bösen. S. 71f.

${ }^{85}$ Siehe Irigaray: Das Geschlecht, das nicht eins ist. S. 191; Hermann Lang: Die Sprache und das Unbewusste. Frankfurt a.M.: Suhrkamp. 3. Aufl. 1998. S. $286 f$.

${ }^{86}$ Suchsland: Julia Kristeva. S. 125.

${ }^{87}$ Kristeva: Powers of Horror. S. 4.

${ }^{88}$ Webster: The Duchess of Malfi. IV.2,231f.

89 " [T] $]$ he madmen themselves have a feminine dimension, since the English tailor is crazed by his assiduous study of new fashions, while the doctor has forfeited his wits by jealousy.' Desmet: 'Neither maid, widow, nor wife'. S. 83.
} 
Einzigartigkeit sich als tödliche Halluzination erweist: In Übereinstimmung mit seiner Überzeugung: "Eagles commonly fly alone: they are crows, daws, and starlings that flock together", isoliert ihn sein Begehren nach Reinheit derart, dass er sogar versucht, sich von seinem Schatten zu trennen. ${ }^{90}$ In völliger Entfremdung von seiner Umwelt wie von sich selbst beginnt er an Lykanthropie zu leiden, das heißt, sich als Wolf zu imaginieren, ironischerweise den Mond anzuheulen und Friedhöfe zu plündern. Als Werwolf wird er zu einem wahrlich einzigartigen Wesen, dem einzigen seiner Spezies, und treibt damit die pervertierte patriarchale 'Logik des Selben' auf die Spitze. Doch diese dekonstruiert sich nicht nur selbst, sondern wird vom Abjekt noch auf einer zweiten Ebene, nämlich durch die Rückkehr der Herzogin als Echo attackiert. ${ }^{91}$

Nicht nur, dass das Echo von Antonio eindeutig als Stimme seiner toten Frau identifiziert wird - " "Tis very like my wife's voice” -, das Echo bestätigt dies auch noch explizit: "Ay, wife's voice".92 Da es durchweg die Wahrheit spricht, ${ }^{93}$ evoziert es das von Cixous postulierte enge Verhältnis von Stimme und Weiblichkeit, wenn auch auf eine etwas andere Art und Weise, als von der französischen Theoretikerin angedacht. Wenn aber die Frau "wholly and physically present in her voice" ist ${ }^{94}$ - und in der Tat ist die Herzogin im fünften Akt nichts außer ihrer Stimme -, wenn man das Auftreten des der Wahrheit verbundenen Echos als Manifestation einer weiblichen Ökonomie am äußersten Rande einer phallischen Gattung lesen kann und wenn sich für Cixous in dieser Stimme immer auch die Stimme der Mutter - und gerade als solche wird die Herzogin im Stück charakterisiert - manifestiert, so gilt es doch zu differenzieren: Während es sich bei Cixous nämlich um die verlorene Mutter der präödipalen Phase im Sinne einer 'Guten Mutter' als 'omnipotent and generous dispenser of love, nourishment and plenitude" handelt, ${ }^{95}$ lassen die dem Echo zugeschriebenen Attribute eine solche Verklärung nicht $\mathrm{zu}$, ist doch die vermeintliche Stimme der Herzogin eindeutig an ein abjektales Konzept von Weiblichkeit gekoppelt.

Einerseits evoziert der marginale Ort, der im fünften Akt der von der Herzogin symbolisierten Weiblichkeit eingeräumt wird, Irigarays These, dass die "Verwerfung, der Ausschluß eines weiblichen Imaginären" die Frau in die Position bringt, "sich nur fragmentarisch beweisen zu können, an den wenig strukturierten Rändern einer herrschenden Ideologie. Als Überbleibsel oder

\footnotetext{
${ }^{90}$ Webster: The Duchess of Malfi. V.2,30f.; ebd. V.2,31ff.

${ }^{91}$ Für eine Interpretation des Echos im Sinne der griechischen Mythologie siehe Desmet: 'Neither maid, widow, nor wife'. S. 85.

${ }^{92}$ Webster: The Duchess of Malfi. V.3,27f.; siehe auch ebd. V.3,44ff.

93 "Do not". Webster: The Duchess of Malfi. V.3,30; "Be mindful of thy safety". Ebd. V.3,33; "O, fly your fate". Ebd. V.3,36; "Thou art a dead thing”. Ebd. V.3,39; "Never see her more". Ebd. V.3,42.

${ }^{94}$ Moi: Sexual/Textual Politics. S. 114.

${ }^{95}$ Ebd. S. 115.
} 
Ausfälle eines Spiegels, der vom (männlichen) 'Subjekt' besetzt wird". 96 Andererseits geht das Stück auf der Inhaltsebene sogar noch darüber hinaus, da es sich um ein mütterliches Echo aus dem Jenseits handelt. Artikulieren kann sich diese mit

So hollow and so dismal, and withal

So plain in the distinction of our words,

That many have supposed it is a spirit

That answers ${ }^{97}$

charakterisierte Stimme nur in den "ruins of an ancient abbey". Sie wird somit explizit an die Sphäre des Übernatürlichen und Schauerlichen, an Gräber, Krankheit und Tod rückgebunden und als ein "thing of sorrow" sowie "a dead thing" mit "[a] very deadly accent" bezeichnet. ${ }^{98}$

Je stärker die von der Herzogin in den ersten vier Akten personifizierte Form von Weiblichkeit auf (und aus) der Handlungsebene des Stücks exorziert wird, umso stärker greift sie im letzten Akt in zirkulären Akten der Subversion phallozentrischer Strukturen auf das Stück über, und zwar sowohl auf inhaltlicher als auch formaler Ebene: Durch die Multiplizität und Unabgeschlossenheit ihrer closing lines sowie durch ihr Wiederauftreten als Echo macht die Herzogin eine eindeutige closure unmöglich. Es scheint, als greife der bereits in der bedroom scene sowie durch die Schwangerschaften offenkundig gewordene 'weibliche Rhythmus' zirkulärer Zeitstrukturen auf die Form des Stücks über, so dass sich dessen Struktur an eine spezifisch weibliche Libido, mit Irigaray sogar an die Morphologie des weiblichen Körpers, rückbinden lässt. ${ }^{99}$ Diese disruptive Form unterminiert nicht nur the 'great man' tragedy (A.C. Bradley), respektive die Tragödie als Gattung und Moment männlicher Subjektkonstitution par excellence, sondern auch die patriarchale Ordnung. ${ }^{100}$ Wenn Haber schreibt, "[that] [t]he Duchess's efforts [. . .] founder (at least in part) on the self-contradictions in which Webster is necessarily involved [. . .] [and that] the play [. . .] points [...] to its own inadequacies", ${ }^{101}$ so sind es gerade die "self-contradictions" und die "inadequacies" der männlichen Diskurs- und Machtform, weniger jedoch

\footnotetext{
${ }^{96}$ Irigaray: Das Geschlecht, das nicht eins ist. S. 29.

${ }^{97}$ Webster: The Duchess of Malfi. V.3,6-9.

${ }^{98}$ Ebd. V.3, 25, 39, 22.

${ }^{99}$ Siehe Haber: "My Body Bestow upon My Women". S. 141; vgl. hierzu Ferdinands tragische Phantasien, die mit der Form des männlichen Körpers und den Rhythmen männlicher Sexualität isomorph sind.

100 "For it is surely remarkable for a 'tragic heroine' to die neither attempting wholly to 'personate masculine virtue,' nor wholly focused on her male partner [. . .], but concerned instead with the mundane comforts of her children; and it necessarily unsettles our notions of 'tragedy' and of 'heroism' when she does so." Haber: 'My Body Bestow upon My Women'. S. 148.

${ }^{101}$ Ebd. S. 147.
} 
diejenigen Websters, die das Stück offensichtlich werden lässt, indem es sich als Tragödie geriert (und auch als revenge tragedy und tragedy of state gelesen worden ist) - oder aber als deren Mimikry im Sinne einer repetition with a difference. Was als Widerspruch innerhalb des Stücks interpretiert werden kann, als fehlende (phallische) Form und Geschlossenheit und in Bezug auf den fünften Akt als "a curious [. . . ] afterthought", ${ }^{102}$ ist das Aufflackern des Semiotischen, das die männliche Transparenz- und Selbstbespiegelungslogik kontaminiert und auf der Ebene der Form wiederholt, was die Herzogin und ihre Manifestation als Echo auf der Inhaltsebene vorführt. Das Andere, das dem Symbolischen Heterogene, das paradoxerweise die Quelle selbst des Symbolischen darstellt, bleibt notwendigerweise präsent, so dass weder das Symbolische noch Websters Tragödie die Spuren ihrer materiellen Basis tilgen können. Und je entschlossener das Stück die Negation dieser mit Kristeva natürlich/materiell/weiblich/mütterlich/semiotisch/abjektalen Basis vorantreibt, umso stärker bringt es genau die negierten Aspekte hervor, aus denen es selbst erst hervorgegangen ist und die es nun zu verschlingen drohen.

Websters Stück dokumentiert somit eindrucksvoll, "[that] the peculiar organisation of abjection actually founds the signifying economy of our culture", ${ }^{103}$ dass Literatur "the ultimate coding of our crises, of our most intimate and most serious apocalypses" 104 darstellt und sich gerade aus der Freude daran speist, "den Raum des Sagbaren durch das Aussprechen des Unaussprechlichen zu erweitern", aus der "Lust am Mitteilen dessen, was vom Gesetz abweicht", und aus der Bereitschaft, sich mit dem Abjekt auseinanderzusetzen. ${ }^{105}$ Dessen wiederholtes, die vermeintlich rationale symbolische Ordnung korrumpierendes Durchscheinen oder Aufblitzen auf formaler wie auch inhaltlicher Ebene könnte somit das von der Kritik (sicherlich nicht ganz zu Unrecht) als grob fehlerhaft bezeichnete, potentiell feministische Ende des Stücks erklären, ${ }^{106}$ das von Bosola in einem vermeintlichen Metakommentar treffend mit "Such a mistake as I have often seen in a play" antizipiert wird. ${ }^{107}$

${ }^{102}$ Siehe Jankowski: Defining/Confining the Duchess. S. 244f.

${ }^{103}$ Smith: Julia Kristeva. S. 149.

${ }^{104}$ Kristeva: Powers of Horror. S. 208.

${ }^{105}$ Gerade in der Literatur führt das "semiotisierende Pulsieren der Triebbasis [. . .] zu 'Verunreinigungen' der symbolischen Strukturen, die immer wieder die Unvollkommenheit und Hinfälligkeit des Subjekts und aller gesellschaftlichen Beziehungen demonstrieren." Suchsland: Julia Kristeva. S. 155.

${ }^{106}$ Potentiell feministisch und inhaltlich insofern problematisch, als das Stück den nicht erbberechtigten Sohn aus einer nicht standesgemäßen Ehe explizit im Namen seiner Mutter erben lässt (Webster: The Duchess of Malfi. V.5,112) und die Existenz eines rechtmäßigen Erben völlig ignoriert.

${ }^{107}$ Webster: The Duchess of Malfi. V.5,94f. 